\title{
SLP-2 interacts with Parkin in mitochondria and prevents mitochondrial dysfunction in Parkin-deficient human iPSC-derived neurons and Drosophila
}

\author{
Alessandra Zanon ${ }^{1, \dagger}$, Sreehari Kalvakuri ${ }^{2, \dagger}$, Aleksandar Rakovic ${ }^{3, \dagger}$, \\ Luisa Foco ${ }^{1}$, Marianna Guida ${ }^{1}$, Christine Schwienbacher ${ }^{1}$, Alice Serafin ${ }^{1}$, \\ Franziska Rudolph ${ }^{3}$, Michaela Trilck ${ }^{3}$, Anne Grünewald ${ }^{3,4}$, \\ Nancy Stanslowsky ${ }^{5}$, Florian Wegner ${ }^{5}$, Valentina Giorgio ${ }^{6}$, \\ Alexandros A. Lavdas ${ }^{1}$, Rolf Bodmer ${ }^{2}$, Peter P. Pramstaller ${ }^{1,7,8}$, \\ Christine Klein ${ }^{3, *}$, Andrew A. Hicks ${ }^{1, *}$, Irene Pichler ${ }^{1, \dagger}$ and Philip Seibler ${ }^{3, \dagger}$
}

${ }^{1}$ Institute for Biomedicine, Eurac Research, Affiliated Institute of the University of Lübeck, 39100 Bolzano, Italy, ${ }^{2}$ Development, Aging and Regeneration Program, Sanford Burnham Prebys Medical Discovery Institute, La Jolla, CA 92037, USA, ${ }^{3}$ Institute of Neurogenetics, University of Lübeck, 23562 Lübeck, Germany, ${ }^{4}$ Molecular and Functional Neurobiology Group, Luxembourg Centre for Systems Biomedicine, University of Luxembourg, L-4367 Belvaux, Luxembourg, ${ }^{5}$ Department of Neurology, Hannover Medical School, 30625 Hannover, Germany, ${ }^{6}$ University of Padova, 35122 Padova, Italy, ${ }^{7}$ Department of Neurology, General Central Hospital, 39100 Bolzano, Italy and ${ }^{8}$ Department of Neurology, University of Lübeck, 23562 Lübeck, Germany

*To whom correspondence should be addressed at: Institute for Biomedicine, Eurac Research, Via Luigi Galvani 31, 39100 Bolzano, Italy. Tel: +39 0471055521; Fax: + 39 0471055599; Email: andrew.hicks@eurac.edu (A.A.H.); Institute of Neurogenetics, University of Lübeck, Maria-GoeppertStraße 1, 23562 Lübeck, Germany. Tel: +4945131018200; Fax: +4945131018204; Email: christine.klein@neuro.uni-luebeck.de (C.K.)

\begin{abstract}
Mutations in the Parkin gene (PARK2) have been linked to a recessive form of Parkinson's disease (PD) characterized by the loss of dopaminergic neurons in the substantia nigra. Deficiencies of mitochondrial respiratory chain complex I activity have been observed in the substantia nigra of PD patients, and loss of Parkin results in the reduction of complex I activity shown in various cell and animal models. Using co-immunoprecipitation and proximity ligation assays on endogenous proteins, we demonstrate that Parkin interacts with mitochondrial Stomatin-like protein 2 (SLP-2), which also binds the mitochondrial lipid cardiolipin and functions in the assembly of respiratory chain proteins. SH-SY5Y cells with a stable knockdown of Parkin or SLP-2, as well as induced pluripotent stem cell-derived neurons from Parkin mutation carriers, showed decreased complex I activity and altered mitochondrial network morphology. Importantly, induced expression of SLP-2 corrected for these mitochondrial alterations caused by reduced Parkin function in these cells. In-vivo Drosophila studies showed a genetic interaction of Parkin and SLP-2, and further, tissue-specific or global overexpression of SLP-2 transgenes rescued parkin
\end{abstract}

\footnotetext{
${ }^{\dagger}$ The authors wish it to be known that, in their opinion, the first three authors should be regarded as joint First Authors and the last two authors as joint Last Authors.

Received: November 16, 2016. Revised: February 24, 2017. Accepted: March 16, 2017

(c) The Author 2017. Published by Oxford University Press. All rights reserved. For Permissions, please email: journals.permissions@oup.com
} 
mutant phenotypes, in particular loss of dopaminergic neurons, mitochondrial network structure, reduced ATP production, and flight and motor dysfunction. The physical and genetic interaction between Parkin and SLP-2 and the compensatory potential of SLP-2 suggest a functional epistatic relationship to Parkin and a protective role of SLP-2 in neurons. This finding places further emphasis on the significance of Parkin for the maintenance of mitochondrial function in neurons and provides a novel target for therapeutic strategies.

\section{Introduction}

Mitochondrial dysfunction has been a longstanding theme implicated in the etiopathogenesis of Parkinson's disease (PD) following observations that mitochondrial toxins can cause parkinsonism in humans and animal models (1). Evidence for a direct relationship between mitochondrial dysfunction and PD originates from an observed $20-30 \%$ decrease of mitochondrial complex I activity in the substantia nigra of patients with sporadic PD $(2,3)$. Notably, complex I deficiencies have also been reported in platelets, lymphocytes, and fibroblasts of PD patients (4-6). Although monogenic forms of PD account for a small percentage of cases, substantial progress towards understanding the role of mitochondria in the disease process has been made by studying their function (7). Parkin (PARK2) mutations are the most frequent known cause of recessively inherited PD. Parkin encodes an E3 ubiquitin ligase that has been implicated in several cellular functions including mitochondrial biogenesis, dynamics, and free radical metabolism (8). In subcellular fractionation studies, endogenous and overexpressed Parkin was predominantly cytosolic, although a portion of endogenous Parkin was suggested to be localized within mitochondria (9). Suppression or knockout of Parkin in Drosophila, Zebrafish, mice or human patient cells leads to severe mitochondrial dysfunction, including decreased ATP production, reduced mitochondrial membrane potential, and an altered mitochondrial morphology (10-13). Interestingly, Parkin knockdown in Zebrafish results in a specific reduction of the activity of the mitochondrial respiratory chain complex I (12). Recently, Parkin in concert with the PD-linked gene PINK1 (PARK6), has been implicated in the degradation of dysfunctional, depolarized mitochondria, a process known as mitophagy (14-16). Parkin translocates in a PINK1-dependent manner from the cytosol to dysfunctional mitochondria and ubiquitinates mitochondrial outer membrane proteins. This in turn induces mitophagy (17). Although causal links between mitochondrial dysfunction and PD have been identified, the molecular mechanisms that lead to the perturbed mitochondrial activity and neuronal degeneration are still widely unknown.

In this study, we have newly identified Stomatin-like protein 2 (SLP-2), an inner mitochondrial membrane protein (18), as a Parkin-binding protein. SLP-2 has been shown to bind the mitochondrial lipid cardiolipin, which is required for mitochondrial membrane formation, and to facilitate the assembly of the respiratory chain complexes. Increased levels of SLP-2 in human Tlymphocytes have been shown to stimulate mitochondrial biogenesis and function (19). However, the function of SLP-2 in neurons has not been extensively studied, especially in a disease context. Here, we demonstrate that SLP-2- and Parkin-depleted cells exhibit similar mitochondrial defects regarding reduced complex I activity, ATP production as well as a fragmented mitochondrial network morphology in neuroblastoma SH-SY5Y cells and induced pluripotent stem cell (iPSC)-derived neurons from PD patients with Parkin mutations. Importantly, we have shown that SLP-2 overexpression rescues the identified mitochondrial dysfunction of Parkin-deficient SH-SY5Y cells and Parkin mutant
iPSC-derived neurons. The rescue effect of SLP-2 was replicated in an in vivo Drosophila model. These findings suggest that SLP-2 and Parkin function in a common pathway, and that induction of SLP-2 can reverse mitochondrial dysfunctions caused by Parkin deficiency in neurons and muscles.

\section{Results}

\section{Parkin interacts with mitochondrial SLP-2}

To identify novel Parkin interactors in mitochondria, we have previously performed Tandem Affinity Purification followed by mass spectrometry of mitochondrial and cytosolic fractions of HEK293T and SH-SY5Y cells (20). Out of nine identified potential interactors in the mitochondrial fractions of both cell lines, the following evidence made SLP-2 the most attractive candidate for further experimental work. As is the case for Parkin, SLP-2 deficiency is likely associated with altered mitochondrial respiration, decreased activity of the respiratory chain complex I, and mitochondrial morphology $(21,22)$. Furthermore, since SLP2 forms a complex with mitofusin-2 (18), a mitochondrial outer membrane fusion protein and a Parkin ubiquitination substrate $(23,24)$, and binds monomeric $\alpha$-synuclein (25), a presynaptic neuronal protein that is genetically and neuropathologically linked to PD, we chose SLP-2 for further investigation of its role in Parkin-induced cellular pathogenesis.

Immunofluorescence staining showed co-localization of SLP-2 with the mitochondrial protein GRP-75 and thus confirmed the mitochondrial localization of SLP-2 (18) (Supplementary Material, Fig. S1). The interaction of Parkin and SLP-2 was further supported by reciprocal co-immunoprecipitation of the endogenous proteins from whole cell lysates extracted from SH-SY5Y cells. Immunoprecipitation with an anti-Parkin antibody and immunoblotting with SLP-2 showed a specific interaction with SLP-2 that was greatly reduced in Parkin knockdown cells. For the reverse experiment, SLP-2 was immunoprecipitated, followed by the detection of Parkin (Fig. 1A). The sensitivity and specificity of the antibodies used in this study to detect Parkin and SLP-2 were validated by expressing lentivirally transduced shRNA antisense constructs for Parkin and SLP-2, which led to a decrease in protein levels (Fig. 1A, Input; Fig. 2A). The knockdown was highly efficient, with more than $95 \%$ reduction in both proteins (Supplementary Material, Fig. S2A and B).

To further investigate the interaction of Parkin and SLP-2, we used the Proximity ligation assay (PLA) to quantitatively assess the binding of endogenous Parkin and SLP-2 in SH-SY5Y cells. Exposure to the mitochondrial uncoupler CCCP significantly increased the positive PLA signal in wild type cells, indicating an increased interaction between the two proteins due to Parkin translocation to the mitochondria; this increase was less pronounced in the case of Parkin knockdown cells as they expressed only a low amount of Parkin (Fig. 1B). To confirm that the interaction of the two proteins occurs at the mitochondria, a co-staining of a PLA experiment for Parkin and SLP-2 with a mitochondrial marker was performed (Fig. 1C). Furthermore, the binding was detected by immunoprecipitation using mitochondrial fractions 

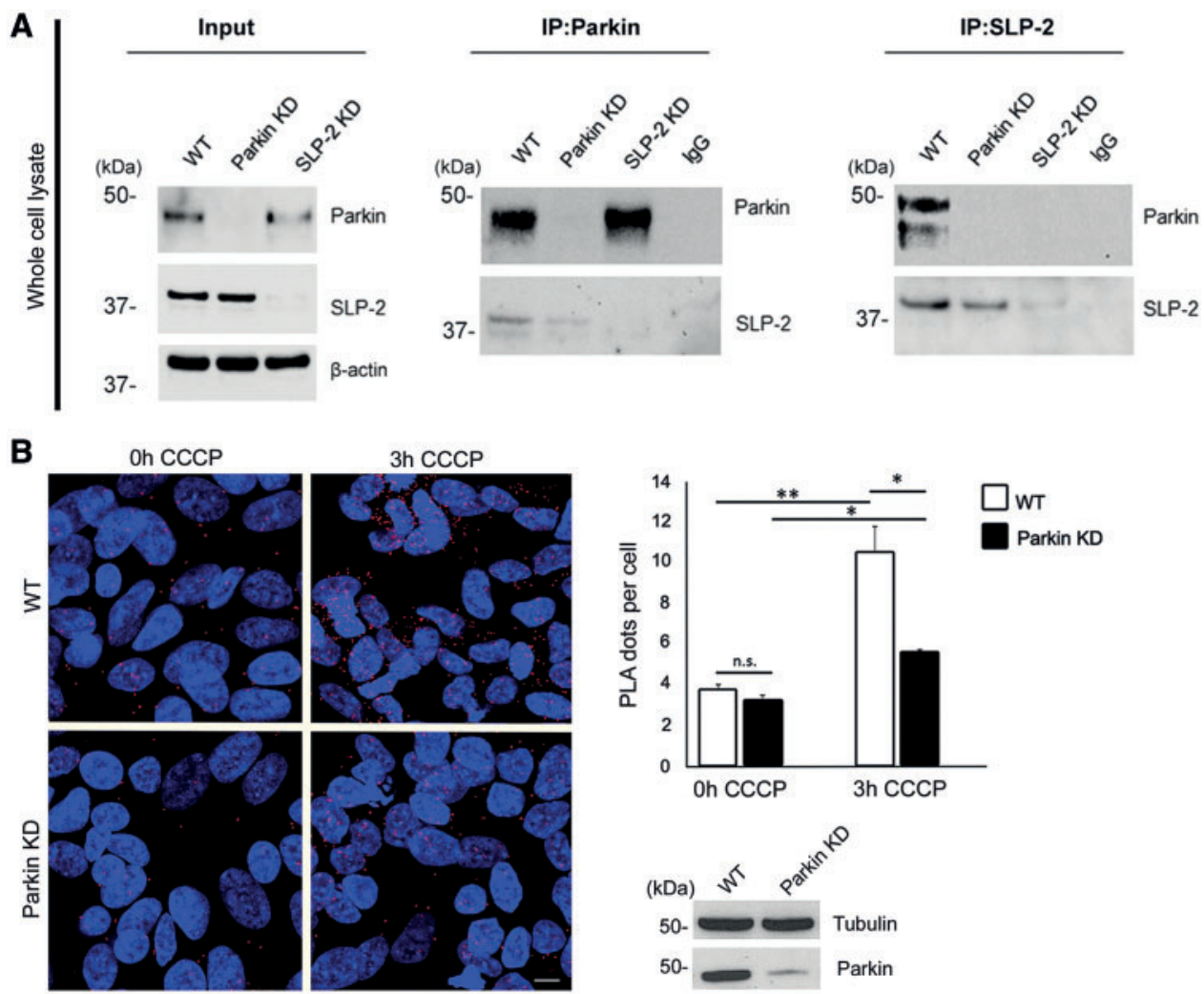

C
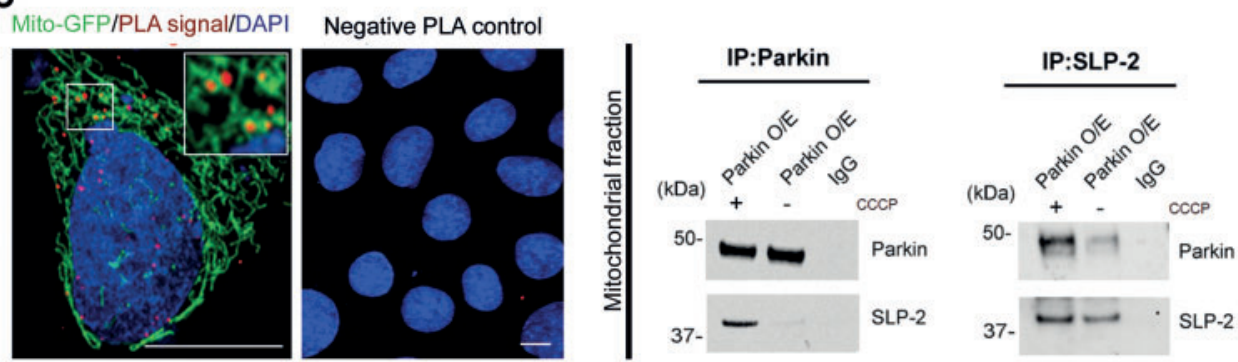

Figure 1. Parkin interacts with mitochondrial SLP-2. (A) Whole cell lysates of untransfected SH-SY5Y cells were subjected to co-immunoprecipitation (IP) with antibodies against Parkin (left panel) and SLP-2 (right panel), followed by Western blotting (WB) of input and IP fractions with the indicated antibodies (the blots were probed consecutively with the antibodies). Cells with knockdown (KD) constructs against Parkin and SLP-2 validate the sensitivity and specificity of the anti-Parkin and anti-SLP-2 antibodies, respectively. IgG was used as negative control for the IPs. Molecular mass markers are in kilodaltons (kDa). (B) SH-SY5Y cells, wild type (WT) and with stable Parkin KD, were processed using the PLA to quantitatively assess the Parkin-SLP-2 interaction under normal culture conditions and after CCCP treatment (3h, $10 \mu \mathrm{M})$. The PLA signal is visualized in red, while DAPI-stained nuclei are shown in blue. Exposure to CCCP increased the PLA signal indicating an augmented interaction between the two proteins. The amount of the increase was higher in WT cells $(2.8 \mathrm{x})$ compared to Parkin KD cells $(1.6 \mathrm{x})$. Two-tailed Students t-test ${ }^{*} \mathrm{P}<0.05 ;{ }^{*} \mathrm{P}<0.01$. n.s. $=$ not significant. Scale $\mathrm{bar}=7.5 \mu \mathrm{m}$. (C) A PLA experiment for Parkin and SLP-2 was co-stained with a mitochondrial marker (green fluorescent protein attached to a mitochondrial leading sequence, mito-GFP) showing a co-localization of the PLA signal with the mitochondria. The specificity of the PLA interaction results was confirmed by performing the experiments with only one of the two primary antibodies. Mitochondrial fractions of SH-SY5Y cells stably overexpressing Parkin, untreated and treated with CCCP, were subjected to IP with anti-Parkin, anti-SLP-2, and anti-IgG antibodies, respectively, followed by WB with the indicated antibodies.

of cells overexpressing Parkin, and an increased amount of coprecipitating SLP-2 was observed upon CCCP treatment (Fig. 1C). Taken together, these data show that Parkin and SLP-2 interact at the mitochondria even under basal culture conditions, an interaction that is enhanced after induction of mitochondrial membrane depolarization.

\section{SLP-2- and Parkin-depleted cells exhibit similar mitochondrial defects}

To assess a functional relationship between SLP-2 and Parkin, we tested whether loss of Parkin affects SLP-2 protein levels by comparing endogenous levels of SLP-2 in two patient fibroblast lines with homozygous or compound-heterozygous Parkin mutations and control lines under basal culture conditions and after exposure to CCCP. Only after $24 \mathrm{~h}$ of treatment we did observe a decrease in SLP-2 levels in the control lines but not in the two Parkin mutant lines. As expected, this effect was not specific for SLP-2, as protein levels of two additional mitochondrial proteins (GRP-75 and AIFM1) also decreased in the control lines after $24 \mathrm{~h}$ treatment due to Parkin-induced mitophagy (Supplementary Material, Fig. S3A). Since Parkin and SLP-2 physically interact, we investigated whether SLP-2 is a substrate of Parkin for ubiquitination or whether depletion of SLP-2 affects Parkin translocation to mitochondria. To test 
A

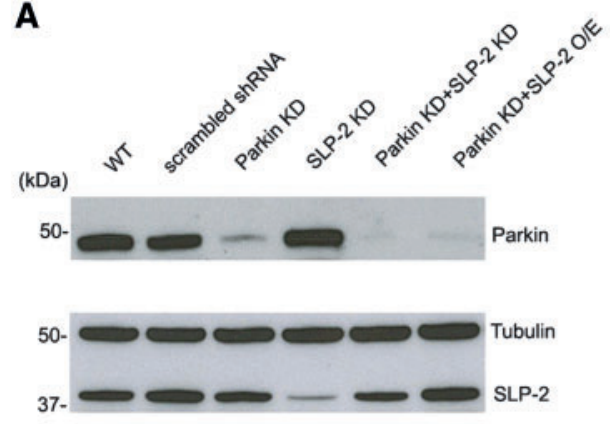

D

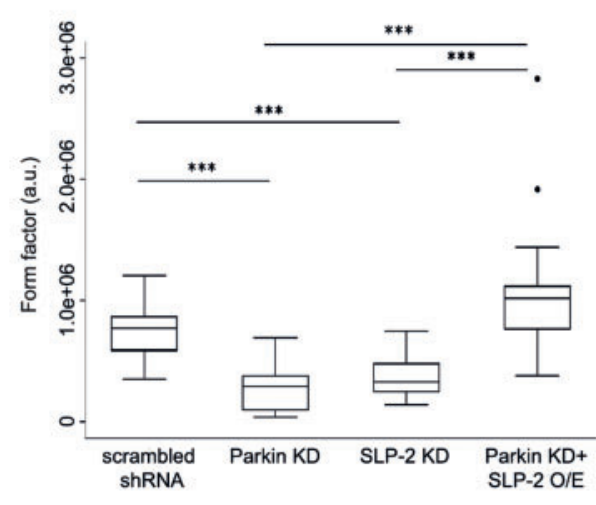

B

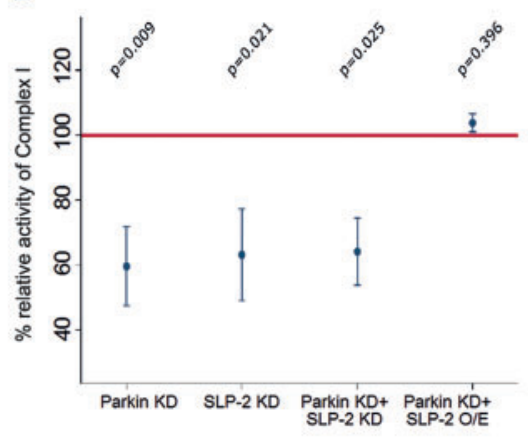

C

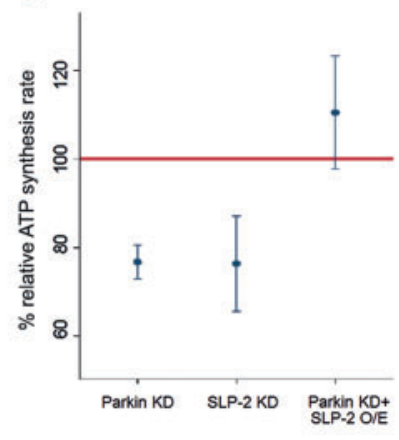

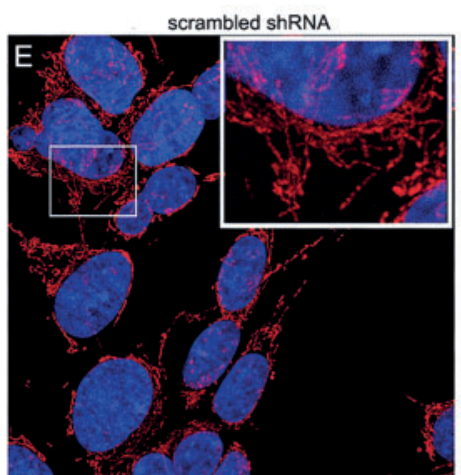

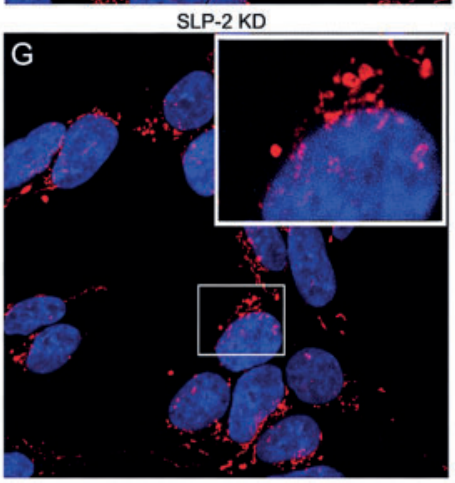

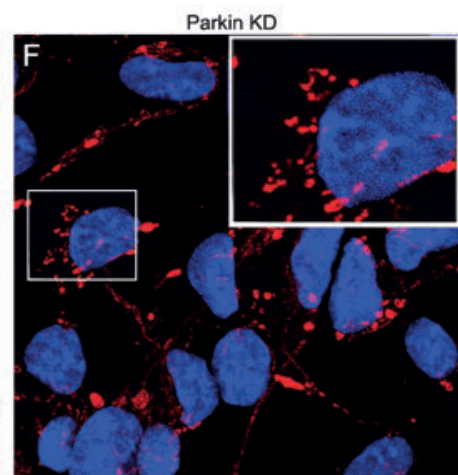

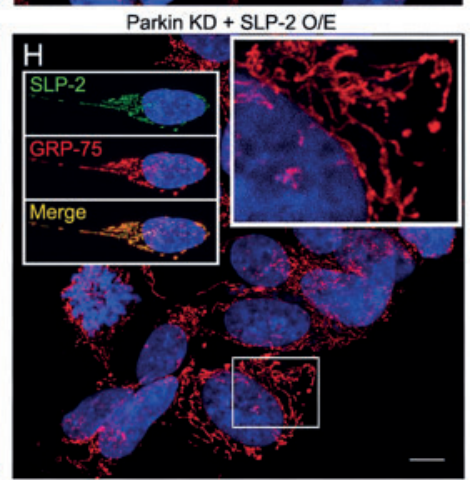

Figure 2. SLP-2 overexpression restores mitochondrial dysfunction in Parkin-deficient SH-SY5Y cells. Complex I activity was determined in mitochondrial fractions of SH-SY5Y cells expressing lentivirally transduced shRNA constructs for Parkin and SLP-2 as well as Parkin-deficient SH-SY5Y cells transduced with a lentiviral construct containing SLP-2. (A) Whole cell lysates of SH-SY5Y cells: scrambled shRNA control, Parkin KD, SLP-2 KD, Parkin + SLP-2 KD, and Parkin KD with SLP-2 overexpression $(\mathrm{O} / \mathrm{E})$, were analyzed by WB with the indicated antibodies. Tubulin served as loading control. (B) Percentage of complex I activity in mitochondrial fractions isolated from SH-SY5Y cells. SLP-2 O/E restored the reduced complex I activity in Parkin KD cells. The horizontal red line indicates the reference activity of complex I in scrambled shRNA control cells and was set to $100 \%$; values are means \pm SEM. Global P-value Kruskal-Wallis test $=0.011$. $P$-values of the pairwise comparisons between each group and the control are shown (adjusted P-value for significance $=0.006$ ). $N=4$, except for SLP-2 KD and Parkin + SLP-2 KD $n=3$. (C) Relative percentages of ATP synthesis rate in SH-SY5Y cells with stable Parkin KD, SLP-2 KD, and Parkin KD with SLP-2 O/E cells compared to control cells. SLP-2 O/E increased the reduced ATP synthesis rate in Parkin KD cells. The horizontal line indicates the reference activity of ATP synthesis in control cells; values are means \pm SEM. Global P-value KruskalWallis test $=0.076 ; n=3$. (D) Mitochondrial network morphology in control, stable Parkin KD, SLP-2 KD, and Parkin KD with SLP-2 O/E SH-SY5Y cells. Global P-value of Kruskal-Wallis test $<0.0001$ (for pairwise comparisons, adjusted $P$-value for significance $=0.004$ ). The degree of mitochondrial branching (form factor) was significantly lower in SLP-2 and Parkin-deficient cells compared to the scrambled shRNA control and compared to the Parkin KD with SLP-2 O/E cells (*** P-value Dunn's test $<0.0001$ ). No statistically significant difference was detected between controls vs Parkin KD with SLP-2 O/E cells and between SLP-2 KD vs Parkin KD. The box plot represents the raw data. Images of 30 randomly selected cells per condition were analyzed. (E-H) Representative examples of images that were analysed are shown. Scale bar $=7.5 \mu \mathrm{m}$.

these hypotheses, we established a cell model of SLP-2 and Parkin-deficient human neuroblastoma SH-SY5Y cells by expressing lentivirally transduced shRNA antisense constructs for SLP-2 and Parkin, respectively (Fig. 2A). We used IP of whole cell lysates from SH-SY5Y cells (overexpressing Parkin, wild type, and with a stable Parkin knockdown, treated with the proteasomal inhibitor MG132) to capture endogenous SLP-2 and to probe for ubiquitin. However, SLP-2 relative ubiquitination was not consistently altered by Parkin expression levels (Supplementary Material, Fig. S3B) compared to immunoprecipitation with mitofusin-2 antibody that we used as a positive control (Supplementary Material, Fig. S3C). Thus, no Parkin-dependent changes in SLP-2 levels and ubiquitination were detected. Further, expression levels of SLP-2 did not impact on Parkin translocation to mitochondria (Supplementary Material, Fig. S3D). 
Since both Parkin and SLP-2 are involved in the overall oxidative phosphorylation activity of mitochondria and more specifically in the activity of complex I $(6,19,21)$, we measured the activity of this complex as a first functional readout (Fig. 2B). In both antisense transduced cell lines, the activity of complex I was reduced compared to cells transfected with control scrambled shRNA. Double knockdown cells did not show a further worsening of the complex I activity. Next, we examined the effect of Parkin or SLP-2 knockdown on ATP production in SH-SY5Y cells (Fig. 2C). We found that Parkin or SLP-2 knockdown resulted in lower ATP synthesis rates compared to control cells. Parkin has been reported to have an important role in the regulation of mitochondrial network morphology, as its deficiency upon knockdown resulted in a truncated and fragmented mitochondrial network in SH-SY5Y cells $(26,27)$. In accordance with these studies, SH-SY5Y cells with a stable knockdown of Parkin showed a fragmented mitochondrial network compared to control cells, and a very similar effect was detected in SH-SY5Y cells deficient in SLP-2 (Fig. 2D-G). Cell viability did not change after SLP-2 knockdown (Supplementary Material, Fig. S3E) showing that the mitochondrial defects are not a downstream result of general cell death.

\section{SLP-2 overexpression restores defects in mitochondria in Parkin-deficient SH-SY5Y cells}

To test whether SLP-2 overexpression can compensate for Parkin deficiency in SH-SY5Y cells, complex I activity was measured after lentiviral SLP-2 transduction in Parkin knockdown cells. Even moderately overexpressed SLP-2, using the promoter of a housekeeping gene (phosphoglycerate kinase) (quantified in Supplementary Material, Fig. S2B), achieved an apparent full recovery of complex I activity (Fig. 2B). Mitochondrial localization of overexpressed SLP-2 was confirmed by immunostaining (Fig. 2H).

The decreased ATP production observed in Parkin knockdown cells increased upon overexpression of SLP-2 in these cells, with rescued production levels often greater than those in controls (Fig. 2C). The alteration of mitochondrial morphology observed in Parkin knockdown cells was also rescued by overexpressing SLP-2 (Fig. 2D-H).

\section{Mitochondrial dysfunction in Parkin mutant iPSC- derived neurons is compensated by overexpression of SLP-2}

In order to verify the mitochondrial effects identified in SHSY5Y cells in a biologically relevant cell type with endogenous Parkin mutations, we reprogrammed skin fibroblasts from a PD patient (iPS-B125) harboring a homozygous mutation (c.1072delT, p.A324fsX110) and two healthy controls (iPS-HFFwt, iPS-L7659-wt) into iPSCs (examples shown in Fig. 3, Supplementary Material, Fig. S4). The iPS-B125 line was generated in collaboration with Dr. Krainc (Northwestern University Feinberg School of Medicine, Chicago, USA) and has been used in a recent study (28). We provide here the data for the characterization of iPS-B125 (Fig. 3). In addition, we employed two lines from healthy individuals that we reported previously (iPSL2134-wt and iPS-L2135-wt) (29). The mutation carrier exhibited onset of clinical symptoms at the age of 43 years, which were characterized as bradykinesia, rigidity, postural instability, and good response to levodopa treatment (30-32). We established one iPSC line per fibroblast line and confirmed the expression of pluripotency markers at both protein and mRNA levels (Fig. 3A and B; Supplementary Material, Fig. S4A and D), the efficient silencing of viral transgenes (Fig. 3C; Supplementary Material, Fig. S4C), and the preservation of a normal karyotype (Fig. 3D; Supplementary Material, Fig. S4B). The presence of the mutation in iPS-B125 was confirmed by direct sequencing (Fig. 3E), and the differentiation capacity of the iPSCs in vitro was determined through the formation of embryoid bodies (Fig. 3F; Supplementary Material, Fig. S4E). The iPSC lines were subsequently differentiated into midbrain type dopaminergic (DA) neurons, and immunofluorescence staining showed neurons co-expressing the neuron-specific marker TUJ1 and TH, the rate-limiting enzyme in the synthesis of dopamine (Fig. 3G). Electric activity is shown for differentiated iPS-B125 and iPSHFF-wt (Fig. 3H; Supplementary Material, Table S1) confirming the state of mature functional neurons.

In line with the results in SH-SY5Y cells, neurons derived from the Parkin mutation carrier iPS-B125 showed a significant reduction of complex I activity as compared to control neurons; this deficit was rescued by overexpression of SLP-2 in iPS-B125derived neurons (Fig. 4A). Differentiation efficiencies were found to be comparable as shown by protein levels of neuronal and DA markers (Fig. 3G; Supplementary Material, Fig. S5A and B).

As observed in Parkin knockdown SH-SY5Y cells, the degree of mitochondrial branching was significantly higher in THpositive neurons derived from iPS-B125 compared to control iPSC-derived neurons, which could also be restored by SLP-2 overexpression in the mutant neurons (Fig. 4B-E). To exclude variability due to reprogramming and differentiation, we examined the mitochondrial network in one additional iPSC line (iPSL3244) that we reprogrammed from a compound-heterozygous Parkin mutation carrier (delEx1; c.924C > T). The iPS-L3244 line was differentiated into DA neurons (Fig. 3G; Supplementary Material, Fig. S4) and TH-positive neurons displayed fragmented mitochondria to a similar degree as found in iPS-B125, an effect that was again compensated by SLP-2 overexpression (Fig. 4B).

\section{Knockdown of Drosophila SLP-2 results in defects in mitochondrial and neuronal function}

To test the in-vivo role of SLP-2, we examined SLP-2 function in the Drosophila model, which is genetically tractable and has been widely accepted as an in-vivo PD model especially to study genes that interact with PINK1 and Parkin (33-35). Interestingly, knockdown of SLP-2 ( 75\% depletion of endogenous SLP-2; Supplementary Material, Fig. S6) caused disruption of mitochondrial network structure accompanied by a significant reduction in ATP levels in energy-demanding adult flight muscles compared to genetically matched controls (Fig. 5A and B). The adult flight muscles are heavily dependent on mitochondrial activity for their energy requirements and therefore quite sensitive to defects in mitochondrial ATP production (36). Consistent with this, flies with reduced SLP-2 exhibited reduced flight ability and wing posture phenotypes ('droopy wings') indicative of muscle dysfunction, as seen with the loss of PD genes like parkin (Fig. 5C and D, Supplementary Material, Fig. S8A). Together, these results suggest that SLP-2 is required for flight muscle integrity and maintenance of bioenergetics. Reduction in neuronal SLP-2 also led to a moderate loss of DA neurons in the PPL1 cluster (Fig. $5 \mathrm{E})$, which is accompanied by a compromised motor function as measured using a climbing assay (Fig. 5F). Pan-neuronal knockdown of SLP-2 also resulted in significant reduction in ATP levels (Supplementary Material, Fig. S7A), suggesting a general requirement of SLP-2 in maintaining mitochondrial bioenergetic function. Further, global knockdown of SLP-2 resulted in partial male 
A

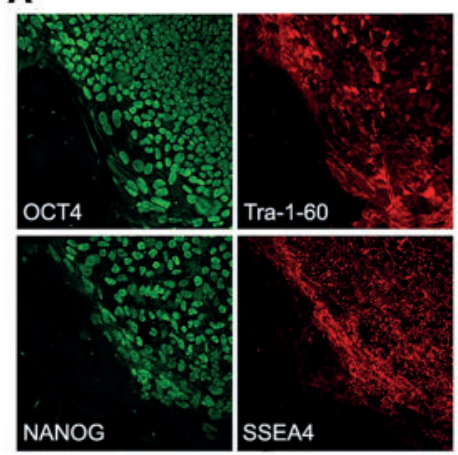

D

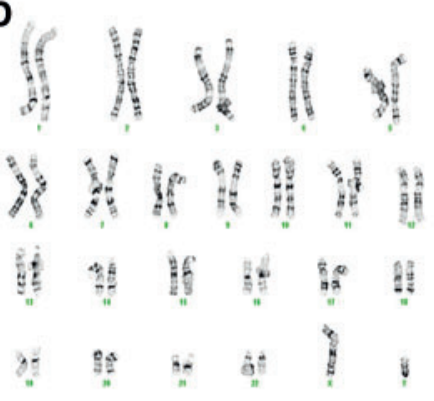

B

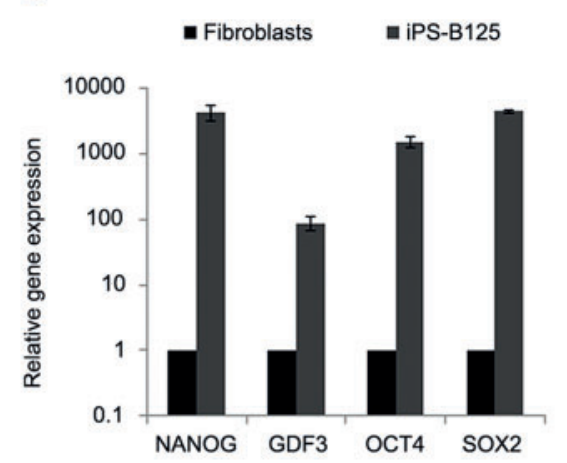

E

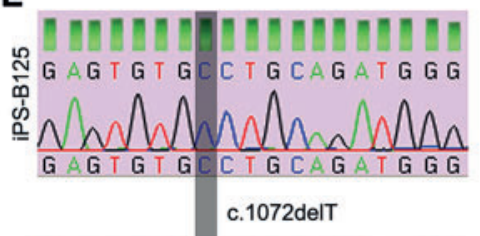

C

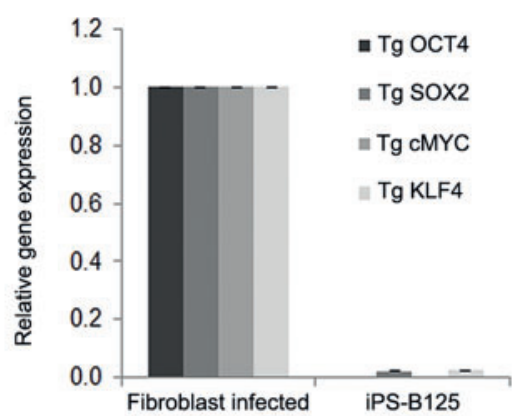

$\mathbf{F}$

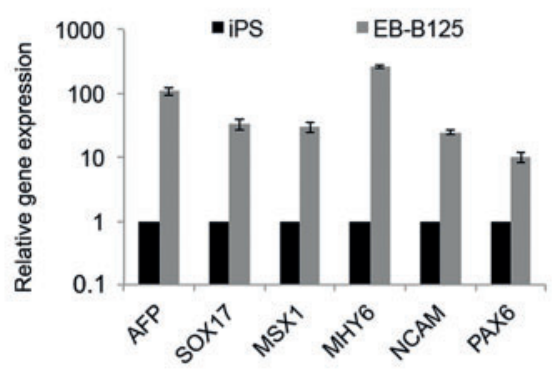

G

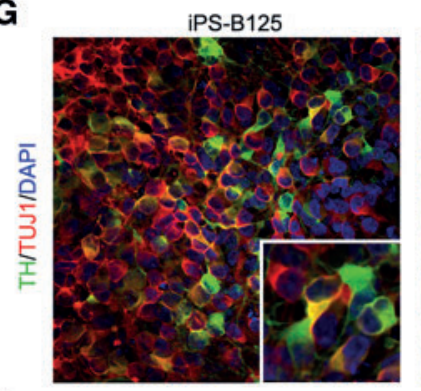

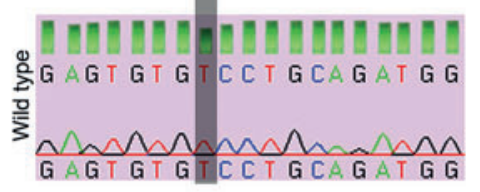
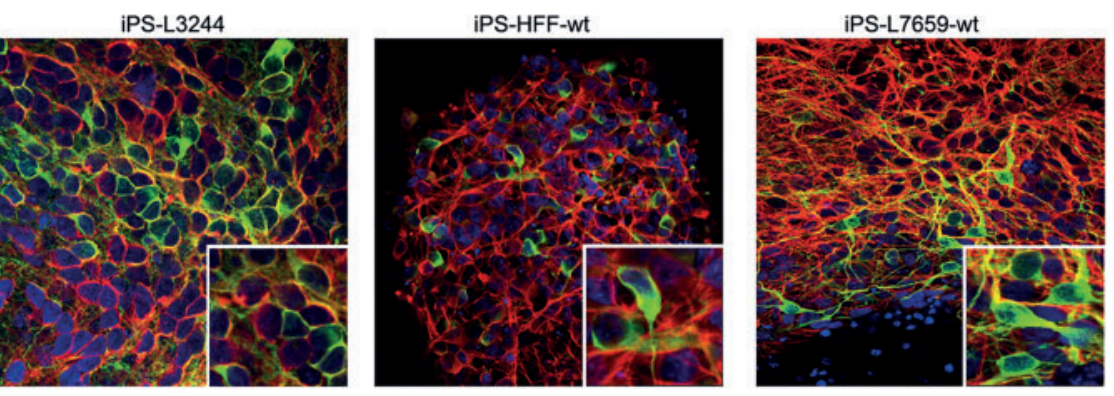

H

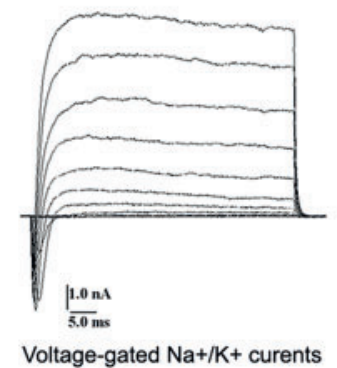

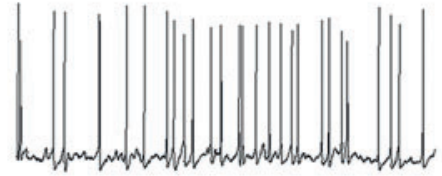

$\left.20 \mathrm{mv}\right|_{\overline{15}}$

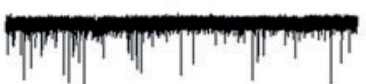

${ }^{20 \mathrm{pA}} \frac{}{5 \mathrm{~s}}$

Spontaneous PSC

Figure 3. Generation of iPSCs and DA neurons. (A-F) Reprogramming of patient-derived skin fibroblasts harboring a Parkin mutation into iPSC line iPS-B125. (A) Immunofluorescence analysis shows the presence of pluripotency markers OCT4, Tra-1-60, NANOG, and SSEA-4 in iPS-B125. (B) Expression levels of endogenous pluripotency markers NANOG, GDF3, OCT4, and SOX2 in fibroblasts and iPS-B125 relative to $\beta$-actin (as loading control) as assessed by quantitative RT-PCR. The values for fibroblasts were set to 1 . The error bars indicate the standard deviation. (C) Residual expression levels of transgenes OCT4, SOX2, cMYC, and KLF4 (relative to $\beta$-actin) were examined by quantitative RT-PCR. The values for the infected fibroblasts (isolated 7 days after infection) were set to 1 . (D) Karyotype of iPS-B125 was normal. (E) Direct sequencing confirmed the Parkin mutation c.1072delT in iPS-B125. (F) RT-PCR analyses of various differentiation markers for the three germ layers (endoderm: AFP, SOX17; mesoderm: MSX1, MHY6; ectoderm: NCAM, PAX6) in iPSCs (iPS) and after 4 days in suspension culture to form embryoid bodies (EB) followed by 7 days in adherent culture. The values are relative to $\beta$-actin as assessed by quantitative RT-PCR and normalized to the parental iPSC line (set to 1 ). (G) Immunofluorescence staining of neuronal cultures derived from iPS-B125, an additional Parkin mutant iPSC line, iPS-L3244 (Supplementary Material, Fig. S4), and the two iPSC control lines, iPS-HFF-wt and iPS-L7659-wt (Supplementary Material, Fig. S4). Staining shows the neuronal marker TUJ1 (red), the DA marker TH (green), and nuclear DAPI (blue). Quantifications were performed for iPS-HFF-wt and iPS-B125 (Supplementary Material, Fig. S5). (H) Neurons from iPS-B125 and iPS-HFF-wt were tested for their electric activity (Supplementary Material, Table S1). They had pronounced voltage-gated $\mathrm{Na}^{+} / \mathrm{K}^{+}$currents, fired evoked multiple and spontaneous action potentials (APs), and showed spontaneous post-synaptic currents (PSC, $n=24$ and $n=9$ cells analysed, respectively). 
A

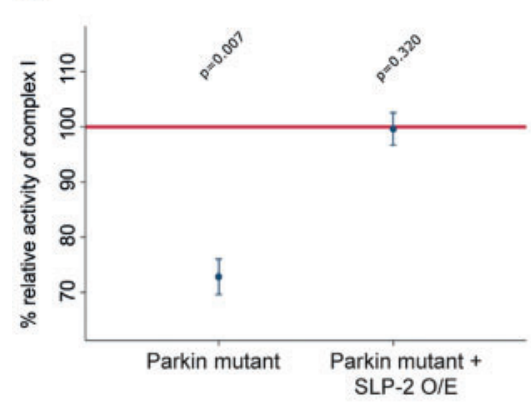

B

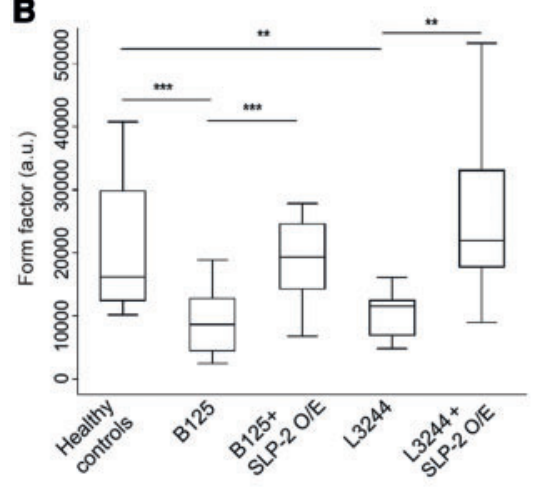

TH
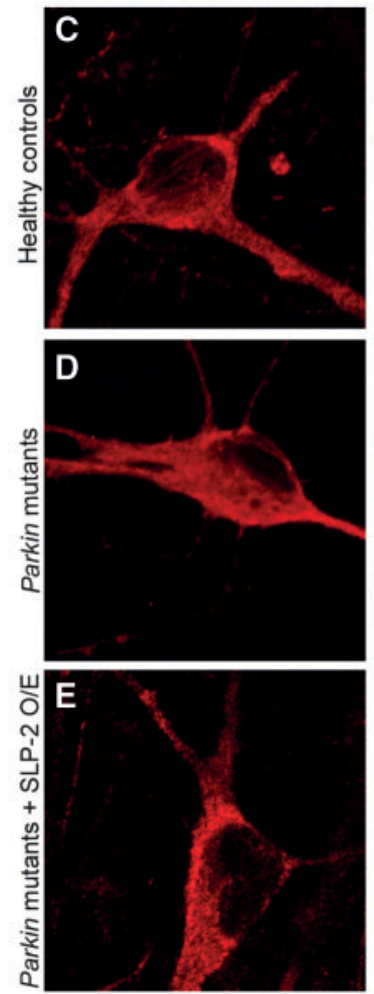

GRP-75
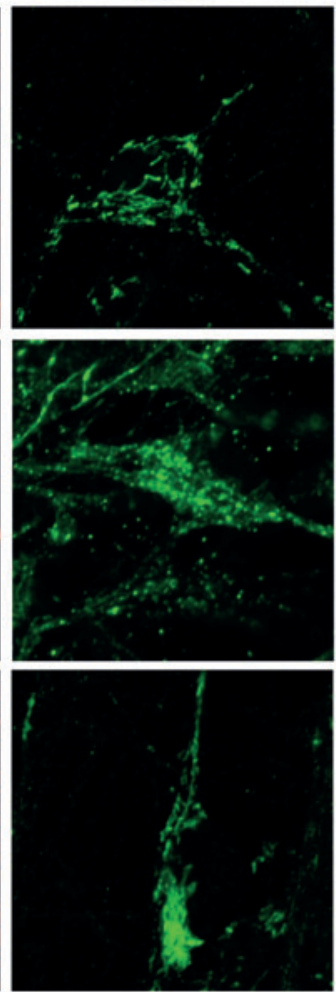

TH / GRP-75 / SLP-2
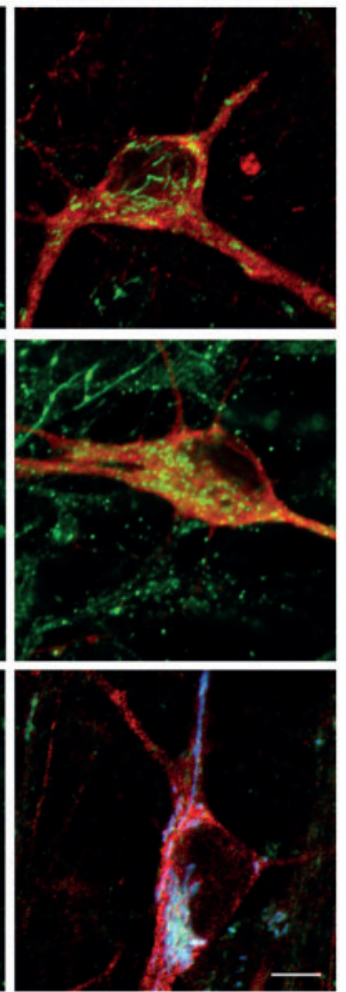

Figure 4. Complex I activity and mitochondrial network morphology in iPSC-derived neurons from patients harboring Parkin mutations. (A) Complex I activity in mitochondrial fractions of neuronal cultures derived from three controls, iPS-B125 and iPS-B125 overexpressing SLP-2. SLP-2 O/E restored the reduced complex I activity in Parkin mutant neurons. The horizontal red line indicates the reference activity of complex I in control neurons; values are means \pm SEM $(P$-value KruskalWallis =0.010; number of observations: control $n=5$, Parkin mutant $n=5$, Parkin mutant + SLP-2 O/E $n=3$ ). Adjusted P-value for significance $=0.0125$. (B) Mitochondrial network morphology is rescued after SLP-2 overexpression in neurons derived from iPS-B125 and iPS-L3244. The degree of mitochondrial branching (mitochondrial form factor) was significantly higher in TH-positive neurons of three healthy control individuals and in the patients' neurons after SLP-2 overexpression compared to the patients harboring Parkin mutations (controls vs iPS-B125: $+57.5 \%, P<0.0001$; controls vs iPS-L3244: $+47.3 \%, P=0.002$; iPS-B125 after SLP- 2 overexpression vs iPSB125: $+59.2 \%, P<0.0001$; iPS-L3244 after SLP-2 overexpression vs iPS-L3244: $+55.8 \%, P=0.004) .{ }^{* *} P<0.0001,{ }^{* *} P<0.01$. Data were derived from three pooled independent experiments and analysed with a random intercept model. Total number of TH-positive neurons analyzed was: controls $=58$, iPS-B125 $=46$, SLP-2 overexpression in iPS-B125 $=25$, iPS-L3244 $=23$, SLP-2 overexpression in iPS-L3244 $=20$. The box plot represents the raw data. (C-E) Immunofluoresence staining of DA neurons for the mitochondrial marker GRP-75 (green), TH (red), and SLP-2 (blue). To clearly visualize differences in the morphology by eye, example images shown were taken from third quartile (upper half of the data) for healthy controls and Parkin mutants overexpressing SLP-2, and from the first quartile (lower half of the data) for the Parkin mutants. Scale bar $=10 \mu \mathrm{m}$.

sterility, which is consistent with the male fertility phenotypes observed with reduction of PINK1 and Parkin activity (37) (Supplementary Material, Fig. S7C).

\section{SLP-2 genetically interacts with Parkin}

Since knockdown of SLP-2 reproduces several phenotypes associated with the loss of Parkin function, we further investigated whether SLP-2 genetically interacts with Parkin in vivo. Reduction in both Parkin and SLP-2 activity led to a further worsening of Parkin-deficiency phenotypes alone (Fig. 5B and F) suggesting that residual SLP-2 activity in parkin mutants maintains partial mitochondrial protection and thus the double mutants produce a more severe phenotype compared to Parkindeficient flies.

\section{Overexpression of SLP-2 rescues Drosophila parkin mutant phenotypes}

The strong genetic interaction between Parkin and SLP-2 and the fact that knockdown of SLP-2 results in similar phenotypes as those observed for Parkin deficiency in human and Drosophila models, led us to hypothesize that boosting SLP-2 levels in vivo would be sufficient to ameliorate loss-of-Parkin phenotypes, as observed in our cell culture experiments. Towards this, we produced SLP-2 overexpression transgenes expressing either the Drosophila SLP-2 ortholog (dSLP-2), or human SLP-2 (hSLP-2), integrated at the same location in the genome (to negate position-dependent changes in transgene expression) (4-5 fold overexpression of SLP-2; Supplementary Material, Fig. S6). Tissue-specific or global overexpression of either Drosophila SLP2 or human SLP-2 rescues Parkin phenotypes, but dSLP-2 offers better rescue compared to hSLP-2 (Fig. 5A-F). Overexpression of SLP-2 transgenes using the indirect flight muscle driver (IFMGal4) is sufficient to rescue the mitochondrial aggregation phenotype seen in parkin null flies (Fig. 5A). SLP-2 overexpression also improves ATP production in parkin mutants and thus boosts flight muscle function as measured by flight and wing posture assays (Fig. 5B-D). Next, we monitored whether SLP-2 overexpression would also rescue phenotypes seen in disease relevant DA neurons. Importantly, overexpression of SLP-2 did rescue the loss of DA neurons observed in parkin null flies and restored the compromised motor function of parkin null 
mutants (Fig. 5E and F). SLP-2 expression in neurons boosts ATP production/bioenergetics, which might be the critical factor underlying the SLP-2-mediated rescue of Parkin phenotypes (Supplementary Material, Fig. S7A). Further, global expression of SLP-2 restores the thoracic indentation and male fertility function in parkin mutants (Supplementary Material, Fig. S7B and C). Thus, our results show that induction of SLP-2 rescues defects in bioenergetics and improves neuronal function in parkin mutant animals.

\section{Discussion}

Loss of Parkin function is one of the most common known causes of PD (38). Previous work has shown that one of the main hallmarks in Parkin-related PD is a decreased activity of complex I of the mitochondrial respiratory chain that has been described in various cell and animal models $(5,6,12)$. Here, we show that the activity of complex I as well as mitochondrial morphology are compromised in Parkin-depleted neuroblastoma SH-SY5Y cells and in iPSC-derived neurons harboring PDcausing Parkin mutations. Most importantly, we identify a novel Parkin interactor in the form of SLP-2, a protein associated to the inner mitochondrial membrane involved in mitochondrial function, biogenesis $(19,21)$, and stress-induced hyperfusion $(22,39)$, that upon overexpression restored the defects in the observed mitochondrial phenotypes of Parkin-deficient neuronal cells. Double knockdown flies showed a genetic interaction between Parkin and SLP-2, and SLP-2 transgenic flies attenuated particular loss of DA neurons, mitochondrial network structure, reduced ATP production, and flight and motor dysfunction. The severity of the double KD in Drosophila might be attributed to differential knockdown of SLP-2 and sensitivity of our in-vivo model as compared to mammalian cells, in which the double knockdown did not result in a further worsening of the parkindependent phenotypes possibly also due to compensatory mechanisms absent in flies.

Our data demonstrate that Parkin interacts with SLP-2 at the mitochondria both in the absence and in the presence of a mitochondrial uncoupler, and that this interaction increases after mitochondrial membrane depolarization. The interaction of Parkin and SLP-2 in the mitochondrial fraction of untreated cells might reflect the localization of a small portion of endogenous Parkin within the mitochondria (9) as well as the fact that a small proportion of mitochondria are depolarized even at basal culture conditions, which might induce some Parkin translocation to the mitochondria (14). Our findings suggest that, unlike mitofusin-2, neither does SLP-2 affect Parkin recruitment to mitochondria, nor does it seem to be consistently ubiquitinated by Parkin.

A T cell-specific knockdown of SLP-2 in mice was shown to be associated with altered mitochondrial respiration, decreased activity of the mitochondrial respiratory complex I and complex II plus III (21), as well as impaired respiratory chain supercomplex formation (40), whereas upregulation of SLP-2 expression translates into enhanced mitochondrial biogenesis and function (19). Similarly, Parkin was found to be involved in mitochondrial respiration, as Parkin-knockout mice exhibited a decreased abundance of mitochondrial proteins involved in oxidative phosphorylation, including several subunits of complexes I and IV, which was accompanied by a reduction in respiratory capacity in striatal mitochondria (11). Our data indicate a significant reduction of complex I activity in Parkindeficient SH-SY5Y cells and neurons differentiated from PD patient-derived iPSCs harboring a homozygous Parkin mutation. These same alterations were recapitulated in SLP-2-deficient cells. Intriguingly, the reduction of complex I activity could be restored by moderate overexpression of SLP-2 in Parkindeficient SH-SY5Y cells and Parkin mutant iPSC-derived neurons. This rescue effect of SLP-2 overexpression was detected also for the phenotype of fragmented and less branched mitochondria that we observed in Parkin-deficient SH-SY5Y cells and Parkin mutant iPSC-derived TH-positive neurons of two patients compared to controls. Mitochondrial morphology in conjunction with Parkin function is currently debated in the literature as to the effects of Parkin mutations. While a greater degree of mitochondrial branching is observed in fibroblasts from Parkin mutation carriers (6), an increased Drp1-dependent mitochondrial fragmentation resulting in less branched mitochondria was seen in SH-SY5Y cells upon Parkin knockdown $(26,27,41)$. Of note, while Drosophila parkin mutants show an abnormally swollen (hyperfused) mitochondrial morphology, parkin knockout mice do not $(10,11)$, but are nevertheless compromised in mitochondrial function. However, mitochondrial morphology examined by electron microscopy in Parkin mutant iPSC-derived neurons from PD patients was described as swollen cristae within the inner mitochondrial membrane with increased perikaryal volume density (42), and others observed a greater proportion of enlarged mitochondria in Parkin mutant compared to control neurons (28). Notably, the latter finding was based on the same Parkin mutant iPSC line that we have used (iPS-B125). In both studies, no mitochondrial form factor was calculated. Moreover, we investigated only TH-positive costained neurons for mitochondrial morphology. Interestingly, different studies reported mitochondrial fragmentation and functional deficits in cultured skin fibroblasts from Parkin or PINK1 mutant patients upon cellular stress $(6,43)$. Therefore, it might be a matter of the presence of reactive oxygen species, which lead to fragmented mitochondria in mammalian cells. Regarding iPSC-derived Parkin mutant DA neurons, it is to be assumed that aged neurons have to deal with a greater amount of reactive oxygen species, which might reflect the situation in our cell model. A recent study has shown that Parkin prevents stress-induced cell death by activating the NF-kB pro-survival pathway. This leads to increased OPA1 levels, a mediator of mitochondrial inner membrane fusion (44), to ensure maintenance of mitochondrial integrity, a protective activity that is not dependent on Parkin translocation to mitochondria and subsequent mitophagy (45). Very recently, SLP-2 was described as part of a new mitochondrial protein complex named SPY which limits the activity of the mitochondrial protease OMA1, resulting in the protection of OPA1 (46). Furthermore, the SPY complex facilitates the cleavage of PINK1 by the mitochondrial protease PARL linking SLP-2 to both Parkin and PINK1. It was suggested that SLP-2, acting as a membrane scaffold, defines the lipid environment for proteolysis and modulates substrate accessibility. Regarding mitochondrial respiration, SLP-2 might promote the compartmentalization of the mitochondrial inner membrane into cardiolipin-enriched microdomains (19), which are required for an optimal assembly and function of the electron transport chain complexes (47). Interestingly, a recent study demonstrated that parkin and pink1 single null flies exhibit a slower turnover of mitochondrial proteins, in particular those of the electron transport chain (48). This suggests that, under physiological conditions, the PINK1-Parkin pathway promotes selective turnover of respiratory chain proteins. The interaction of Parkin with SLP-2 might be essential for a 
A

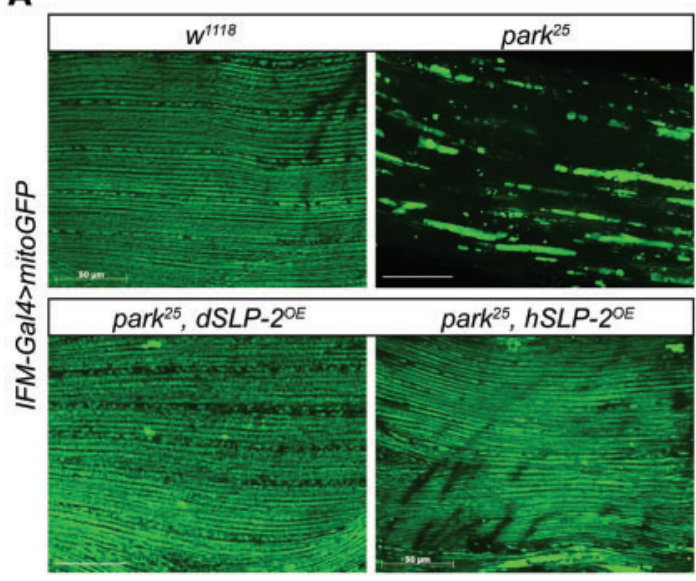

C

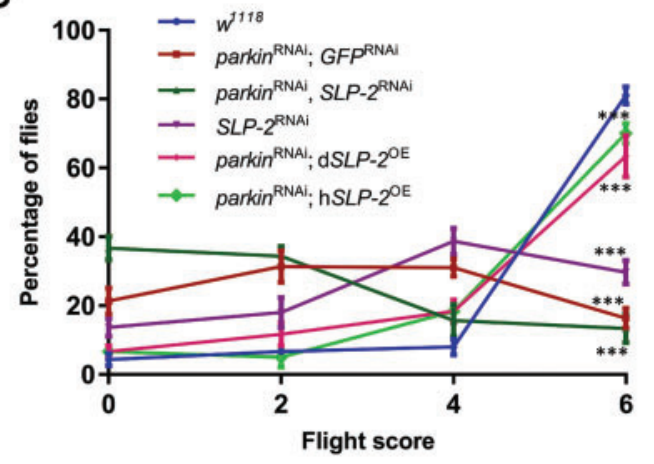

E

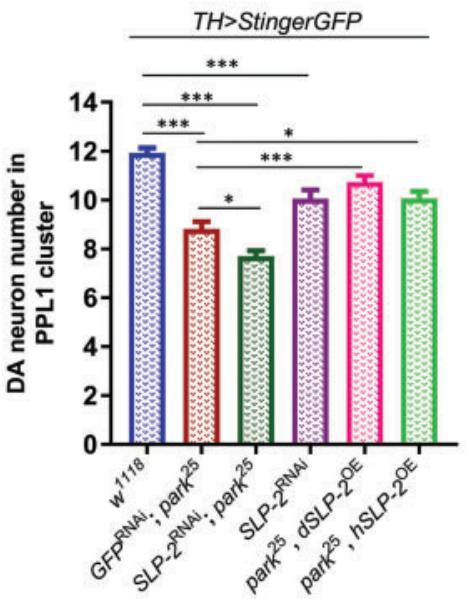

B

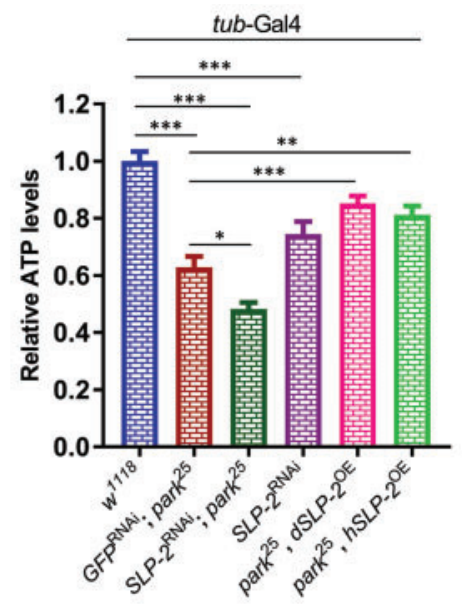

D

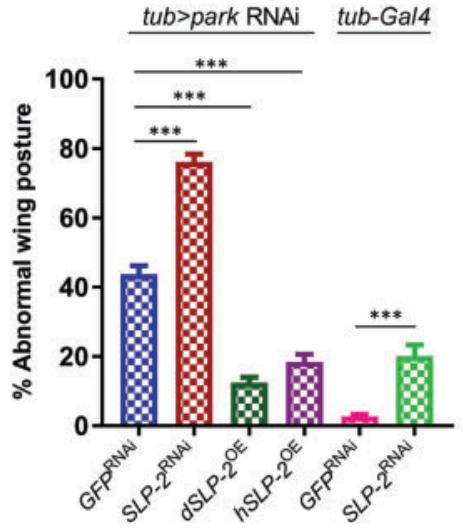

F

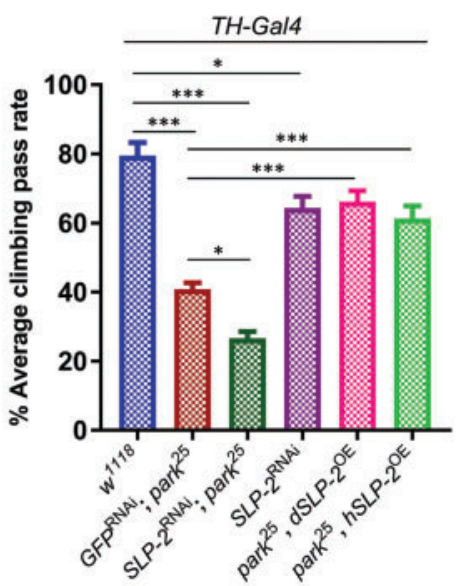

Figure 5. SLP-2 genetically interacts with Parkin and rescues parkin mutant phenotypes when overexpressed in the Drosophila model. (A) Mitochondrial morphology visualized by mitoGFP transgene driven by IFM-Gal4 in adult indirect flight muscles of wild type $\left(w^{1118}\right.$ ) and parkin null (park ${ }^{25}$ ) flies alone or in combination with Drosophila SLP-2 (dSLP-2) or human SLP-2 (hSLP-2) overexpression transgenes. Indirect flight muscles from thoraces of 3-days old flies were dissected to record mitoGFP pattern. Scale bar $=50 \mu \mathrm{m}$. (B) Measurements of ATP levels from thoracic muscles of 5-days old flies expressing transgenes in all tissues under the control of tub-Gal4. The relative levels of ATP were determined by dividing the luminescence by the total protein concentration and normalized to wild-type flies. (C) Flight ability of wild type $\left(w^{1118}\right)$, parkin and SLP-2 RNAi fly lines with or without SLP-2 transgenes expressed under the control of tub-Gal4. 1-week old flies were used to perform the flight assay. A minimum of 100 flies were tested each time in two independent experiments. Flight score of 6 indicates a wild type flight function, score of 0 represents a flightless phenotype. (D) Quantification of abnormal wing posture phenotype in wild type, parkin and SLP-2 RNAi flies with or without SLP-2 transgenes. The percentage of flies displaying abnormal wing posture was determined in 2-weeks old F1 males. (E) Quantification of DA neurons in PPL1 cluster. The numbers of DA neurons in the PPL1 cluster visualized by TH > StingerGFP on the posterior side of the brain were counted. (F) Measurement of motor function using climbing assay. 4-weeks old males aged at $25^{\circ} \mathrm{C}$ were subjected to climb an $8 \mathrm{~cm}$ mark, and the percentage of flies that crossed this mark in $10 \mathrm{sec}$ was determined. A minimum of 100 flies were tested in repeated trails as described in the methods section. Transgenes were expressed under the control of TH-Gal4. Statistical differences were calculated by oneway ANOVA followed by Tukey's post hoc test to correct for multiple comparisons. Data is plotted as $\pm \mathrm{SEM} .{ }^{* * *} \mathrm{P} \leq 0.001,{ }^{* *} \mathrm{P} \leq 0.01,{ }^{*} \mathrm{P} \leq 0.05$. 
mechanism that includes compartmentalization of the inner mitochondrial membranes into cardiolipin-enriched microdomains and the selective turnover of respiratory chain proteins, thereby affecting respiratory chain and ultimately DA neuron function. Our data on the rescue of functional defects seen in Parkin-deficient neuronal cells and Drosophila models by SLP-2 overexpression places SLP-2 downstream or in parallel to Parkin. Notably, overexpression of Parkin rescues motor dysfunction and flight muscle phenotypes seen in SLP-2 deficient flies (Supplementary Material, Fig. S8).

In conclusion, our study shows that Parkin and SLP-2 are able to interact functionally and that this might promote optimal activity of mitochondrial respiratory chain complex I and mitochondrial integrity in cultured cells. We infer from these experiments that under normal conditions, the interaction of Parkin with SLP-2 has a stabilizing role with respect to mitochondrial function. Importantly, we demonstrate in vitro and in vivo that both molecular and physical phenotypes caused by Parkin loss including DA neuron degeneration, can be rescued by SLP-2 overexpression. These findings reveal a novel functional mechanism whereby SLP-2 may aid Parkin in mitochondrial quality control and vice versa, and highlight the importance of the interaction between these two proteins in maintaining robust mitochondrial function. Several attempts have been made to rescue Parkin or PINK1 deficiency by pharmacological compounds in different model systems. Our data suggest that overexpression of SLP-2 might, in the absence of Parkin, compensate for this deficiency, restoring vital mitochondrial and neuronal function. Thus, SLP-2 provides a new target for the development of rescue strategies by specifically targeting the mitochondrial dysfunction in neurons implicated in the etiopathogenesis of PD.

\section{Materials and Methods}

\section{Patients}

Skin biopsies were collected from Parkin mutation carriers and healthy controls, who do not carry any mutations in known PD genes. The study was approved by the ethics committee of the University of Lübeck, and all participants gave written informed consent prior to their participation in the study. Phenotypic and genotypic data are listed in Supplementary Material, Table S2 (further clinical details were published earlier $(30,32))$.

\section{Cell culture}

Human neuroblastoma cells (SH-SY5Y, ATCC CRL-2266), 293FT cells (Life Technologies), and human skin fibroblasts were cultured in Dulbecco's modified Eagle's Medium (DMEM, Sigma) supplemented with $10 \%$ fetal bovine serum and $1 \%$ penicillinstreptomycin (Life Technologies). Human iPSCs were cultured on irradiated mouse embryonic feeder (MEF) cells (GlobalStem) in iPSC medium: DMEM/F12 (StemCell Technologies) with $20 \%$ knockout serum, $1 \%$ L-glutamine, $1 \%$ nonessential amino acids, $0.2 \%$ 2-mercaptoethanol, 10ng/ml Fibroblast Growth Factorbasic (Millipore), and 1\% penicillin-streptomycin. IPSC colonies were passaged enzymatically using Collagenase type IV (Life Technologies) once a week. To depolarize the mitochondrial membrane potential, m-chlorophenylhydrazone (CCCP, $10 \mu \mathrm{M}$, Sigma) was used. To inhibit the ubiquitin proteasome system, MG132 (10 $\mu$ M, Sigma) was used.

\section{Cellular reprogramming, differentiation, and characterization}

The generation of iPSCs from primary human dermal fibroblasts of Parkin mutant and control lines (Supplementary Material, Table S2) was performed by transduction of OCT4, SOX2, cMYC, and KLF4 using four retroviral pMIG vectors as we have described previously (49). Karyotype analysis was performed at the Department of Human Genetics (University of Lübeck, Germany) and Cell Line Genetics (Madison, USA) using standard protocols. The evaluation of in vitro spontaneous differentiation of iPSCs to form embryoid bodies was performed as described previously (50). The direct differentiation of iPSCs into DA neurons was conducted as described previously (51), with minor modifications. In brief, iPSC colonies were disaggregated into single cells and replated onto matrigel-coated dishes (BD) in MEF-conditioned medium, supplemented with ROCK inhibitor Y-27632 (EMD Biosciences). Differentiation was started once iPSCs reached a confluence of $100 \%$ by adding knockout serum replacement (KSR) medium supplemented with SMAD pathway inhibitors SB431542 (SB, Tocris Bioscience) and LDN-193189 (LDN, Stemgent). On days 1 to 5, KSR medium was added to the cells in the presence of SB, LDN, Recombinant Human Sonic Hedgehog (SHH, R\&D System), Recombinant Human FGF-8a (FGF8, R\&D System) and Purmorphamine (Pu, Stemgent). The Wnt pathway activator molecule CHIR99021 (Stemgent) was included from days 3 to 12. During days 6-10 of differentiation, increasing amounts of Neurobasal medium (Life Technologies) plus SM1 neuronal supplement (StemCell Technologies) was added to the KSR medium $(25 \%, 50 \%, 75 \%)$ and upon day 7, SHH, FGF8a, and Pu were withdrawn. On day 11, maturation of DA neurons was initiated by adding Recombinant Human BDNF (Peprotech), ascorbic acid (Sigma), Recombinant Human TGF- $\beta 3$ (Peprotech), cyclic-AMP (EnzoLifescience) and DAPT (Tocris, 10 $\mu \mathrm{M})$ as previously reported (51). On day 20 of differentiation, cells were passaged en bloc, and neuronal precursors were terminally differentiated for 50-70 days.

\section{Electrophysiology}

Eletrophysiological measurements were conducted as previously described (52). Whole cell patch clamp experiments were performed under optical control (inverted microscope, Zeiss) at room temperature. Currents were low-pass filtered at $2.9 \mathrm{kHz}$, digitized at $10 \mathrm{kHz}$ using an EPC-10 amplifier (HEKA) and analyzed with Patch Master (HEKA).

\section{Western blot}

SDS PAGE was performed using NuPAGE 4-12\% Bis-Tris gels (Life Technologies). After electrophoresis, proteins were transferred onto a nitrocellulose membrane (Biorad) and probed with the following antibodies: mouse anti-Parkin (Cell Signaling), mouse anti-SLP-2 (Abcam), mouse anti- $\beta$-actin (Sigma), mouse anti-tubulin (Abcam), mouse anti-ubiquitin (Cell Signaling), mouse anti-MFN2 (Abcam), mouse anti-AIFM1 (Abcam), rabbit anti-GRP-75 (Abcam), mouse anti-MTCO2 (Abcam); rabbit anti-TH (Calbiochem), rabbit anti-NSE (Polysciences), and rabbit anti-GAPDH (Cell Signaling). For densitometric analyses, ImageJ $1.45 \mathrm{~s}$ was used. 


\section{Co-immunoprecipitation}

Co-immunoprecipitation assays were performed in whole cell lysates and mitochondrial fractions of SH-SY5Y cells. Cells were resuspended in lysis buffer $(150 \mathrm{mM} \mathrm{NaCl}, 50 \mathrm{mM}$ Tris- $\mathrm{HCl}, \mathrm{pH}$ 7.6, 1\% NP-40, 0.1\% SDS), supplemented with protease and phosphatase inhibitors. Samples were precleared by incubation with protein A agarose beads (Roche Diagnostics). Beads were removed by centrifugation and the samples incubated overnight at $4{ }^{\circ} \mathrm{C}$ with rabbit anti-Parkin (Abcam), rabbit anti-SLP-2 (Abcam), rabbit anti-MFN2 (Sigma) or control IgG antibodies. Protein A agarose beads were added to the samples followed by incubation on a rotator for $2 \mathrm{~h}$. The beads were washed, resuspended in loading buffer containing DTT (Life Technologies), and finally, the proteins were released from the beads by incubation at $95^{\circ} \mathrm{C}$ for $5 \mathrm{~min}$. After centrifugation, the samples were analyzed by Western blotting. For the ubiquitination assays, SH-SY5Y cells were lysed in denaturing lysis buffer (1\% SDS) and sonicated for $10 \mathrm{sec}$ at 10 microns amplitude. Protein extracts were cleared by centrifugation and diluted 1:10 with non-denaturing lysis buffer.

\section{Immunostaining and PLA}

For immunocytochemical analysis, SH-SY5Y cells, iPSCs and neurons were fixed in $4 \%$ formaldehyde and incubated with primary antibodies (mouse anti-SLP-2 (Abcam), rabbit anti-GRP-75 (Abcam), rabbit anti-OCT4 (Abcam), mouse anti-Tra-1-60 (Millipore), rabbit anti-Nanog (Stemgent), mouse anti-SSEA-4 (Millipore), mouse anti-TUJ1 (Covance), rabbit anti-TH (Calbiochem)). Images were acquired using confocal microscopes (Leica SP8-X; Zeiss LSM710) and the respective software tools LAS-X and ZEN as well as ImageJ and Adobe Photoshop CS6.

The mitochondrial network in SH-SY5Y cells and iPSCderived neurons was stained with an anti-GRP-75 antibody. By means of Image $1.45 \mathrm{~s}$, raw images were binarized, mitochondrion area and outline were measured, and the form factor (defined as $[\mathrm{Pm} 2] /[4 \pi \mathrm{Am}]$ ), where $\mathrm{Pm}$ is the length of the mitochondrial outline and Am is the area of the mitochondrion (13), was calculated. The form factor allows quantifying the degree of branching of the mitochondrial network.

To visualize the interaction between endogenous Parkin and SLP-2 in fixed cells, we also used the PLA ("Duolink" kit, Olink Bioscience (53)). Briefly, cells were fixed and incubated with primary antibodies and with the oligonucleotide-conjugated secondary antibodies, followed by the addition of the ligation solution, so that the two oligonucleotides hybridized and joined to a closed circle when they were in close proximity $(<40 \mathrm{~nm})$. Next, the amplification solution was added, initiating a rollingcircle-amplification reaction to generate a concatemeric DNA strand onto which the fluorescent detection probes subsequently hybridized resulting in the tagging of areas where the two proteins are in close proximity. Samples were observed under a Leica SP5 confocal microscope.

\section{Quantitative analysis of RNA expression levels}

Total RNA was extracted from fibroblasts and iPSCs using the RNA easy protect kit (Qiagen). RNA was reverse-transcribed into cDNA with the Super Script First-Strand Synthesis System (Life Technologies). Quantitative real time PCR was performed with SYBR Green (Roche Diagnostics) on the LightCycler 480 system (Roche Diagnostics). Respective primer sequences are listed in
Supplementary Material, Table S3. All measurements were performed in duplicates per PCR run.

\section{Transient and stable transfections}

For the generation of stable cell lines, the lentiviral pLKO.1 vector (Addgene) was used to express shRNA for human SLP-2 (Sense: ccggGGCTAAAGCTGAAGCTATTTTctcgagAAAATAGCTT CAGCTTTAGCCtttttg) and Parkin (Sense: ccggCCAGTAGCTTTG CACCTGATTctcgagAATCAGGTGCAAAGCTACTGGtttttg). The human SLP-2 and Parkin coding sequences were cloned into the lentiviral pER4 vector with neuron-specific PGK promoter (49). All constructs were validated by sequencing. Virus production was conducted according to standard protocols (54). SH-SY5Y cells and iPSC-derived neurons were infected with a multiplicity of infection of one to three. For lentiviral infection control we used control shRNA Lentiviral Particles-A (sc-108080, Santa Cruz Biotechnology).

\section{Assessment of mitochondrial function}

Mitochondria were isolated from SH-SY5Y cells and iPSCderived neurons (55). In brief, cells were harvested and homogenized in buffer containing $250 \mathrm{mM}$ sucrose, $10 \mathrm{mM}$ Tris, $1 \mathrm{mM}$ EDTA (pH 7.4), and protease and phosphatase inhibitors (Roche Diagnostics). The homogenate was centrifuged twice at 1,500 $\mathrm{x}$ $\mathrm{g}$ for $10 \mathrm{~min}$. The supernatant containing intact mitochondria was centrifuged at $8,400 \mathrm{x} g$ for $10 \mathrm{~min}$, and the mitochondriaenriched pellet used for further analysis. Complex I activity was determined by measuring the oxidation of NADH to $\mathrm{NAD}^{+}$at $340 \mathrm{~nm}$ as previously described (56). Citrate synthase was assayed spectrophotometrically according to a published protocol (57). The final data were expressed as ratios of complex I/citrate synthase. As a normalization step, we computed within each experiment the percentage activity of complex I data (the control cell line was set to $100 \%$ ). Cellular ATP synthesis rates were measured as we have shown previously (13). As for complex I activity data were finally expressed as the percentage of ATP synthesis of control cells.

\section{Drosophila stocks}

Flies were raised on standard cornmeal food at $25^{\circ} \mathrm{C}$ unless otherwise mentioned. To generate dSLP-2 and hSLP-2 transgenic flies, cDNAs of Drosophila and human SLP-2 were cloned separately into pUAST-attB vector and the constructs were injected into attP2 (site-specific integration) flies by BestGene Inc. SLP-2 $\left(48124^{\mathrm{GD}}\right)$ and parkin $\left(104363^{\mathrm{KK}}\right)$ RNAi were obtained from VDRC stock center. IFM-Gal4, UAS-mitoGFP, UAS-StingerGFP, tubGal4, TH-Gal4 and SLP-2 deficiency stocks were obtained from Bloomington stock center. parkin null allele $\left(\right.$ park $^{25}$ ) and UASparkin were kind gifts from Leo Pallanck.

\section{ATP measurement}

Measurements of ATP were performed using a luciferase assay as described previously (58). Five Thoraces from 5-day-old flies were collected after removing wings and legs and homogenized in $100 \mu$ l extraction buffer ( $100 \mathrm{mM}$ Tris and $4 \mathrm{mM}$ EDTA, pH 7.8) containing $6 \mathrm{M}$ guanidine- $\mathrm{HCl}$ followed by rapid freezing in liquid nitrogen. The samples were boiled for $5 \mathrm{~min}$ and cleared by centrifugation at $14,000 \mathrm{x}$ g. Similarly, 10 heads were processed from 2-week-old flies, and ATP levels were determined. 
Relative levels of ATP were determined by dividing the luminescence by total protein concentration as measured by BCA method (Pierce).

\section{Wing posture, fertility, and behavioral assays}

For abnormal wing posture analysis, F1 male flies were collected and aged at $29^{\circ} \mathrm{C}$ for 2 weeks. The percentage of flies with abnormal wing posture (held-up or drooped) was calculated $(58,59)$. For each genotype, 200 flies were scored for their wing posture phenotype in two independent experiments. Quantification of thoracic indentation was done by examining the thoracic indentations of 2-week-old flies under the light microscope, and the percentage of flies with indentations on the thorax was determined. A total of 100 flies were counted per genotype. Flight assay was carried out as described previously (60). Briefly, 1-week-old flies were released slowly in to a clear large box with a light source at the top. The flies that flew towards the light source were given the highest score (score of 6) compared to horizontally flown (score of 4), downwardly flown (score of 2), and flightless flies (score of 0). A minimum of 100 flies were tested each time in two independent experiments. Motor function of each genotype is quantified as described previously $(44,61)$. Male fertility function is determined by crossing single F1 males to five virgin females and quantifying the resulting F2 progeny. A minimum of 20 males were tested for each genotype.

\section{DA neuron counting}

Brains were dissected in PBS and fixed in $4 \%$ formaldehyde for $30 \mathrm{~min}$ followed by three washes in PBS each for $15 \mathrm{~min}$. The images of DA clusters as visualized by TH-Gal4 > UASStingerGFP were obtained by Zeiss apotome microscope. The numbers of DA neurons in PPL1 cluster on the posterior side of the brain were determined by Zeiss imaging software and ImageJ (62).

\section{Statistical analysis}

Raw data were initially plotted for visual inspection; in box plots, the upper and lower adjacent values were calculated as defined by Tukey (63). The normality of the data distribution was assessed using the Skewness-Kurtosis test. Differences between groups for the relative activity of complex I, the ATP synthesis rate and the mitochondrial form factor in SH-SY5Y cells and iPSC-derived neurons were assessed using the nonparametric Kruskal-Wallis test, due to the departure from normality of the outcomes. Post-hoc pairwise comparisons were performed using a Dunn's test; we used different adjusted Pvalue thresholds to correct for multiple comparisons, depending on the number of groups compared to the control group $(P=0.006$, four groups; $P=0.008$, three groups; $P=0.0125$, two groups). In iPSC-derived neurons, the mitochondrial form factor data generated from three independent experiments were pooled and analysed using a random intercept model (64). The genetic status (e.g. scrambled shRNA control, Parkin or SLP-2 knockdown) was included in the model as a fixed effect, while the experiment was considered as a random effect. The random intercept model was fitted using the command xtmixed implemented in Stata 13. All analyses were performed using Stata 13 (StataCorp. 2013. Stata Statistical Software: Release 13. College Station, TX: StataCorp LP).

\section{Supplementary Material}

Supplementary Material is available at HMG online.

\section{Acknowledgements}

We are indebted to the PD patients who have participated in this study. We would like to thank Roberto Melotti for statistical advice.

Conflict of Interest statement. Alessandra Zanon, Sreehari Kalvakuri, Aleksandar Rakovic, Luisa Foco, Marianna Guida, Christine Schwienbacher, Alice Serafin, Franziska Rudolph, Anne Grünewald, Nancy Stanslowsky, Florian Wegner, Valentina Giorgio, Alexandros A. Lavdas, Rolf Bodmer, Andrew A. Hicks, Irene Pichler, Philip Seibler declare no competing interests. Peter P. Pramstaller received honoraria for serving on scientific boards and speaking from Novartis, Boehringer, GlaxoSmithKline, Lundbeck and UCB. Christine Klein serves as medical advisor to Centogene and received an honorarium for speaking by Biogen Idec.

\section{Funding}

Ministry of Health and Department of Educational Assistance, University and Research of the Autonomous Province of Bolzano; German Research Foundation [grant number KL1134/ 11-1, KL1134/16-1, SE2608/2-1]; Hermann and Lilly Schilling Foundation; Innovative Medicines Initiative ['The research leading to these results has received support from the Innovative Medicines Initiative Joint Undertaking under grant agreement number 115439, resources of which are composed of financial contribution from the European Union's Seventh Framework Programme (FP7/2007-2013) and EFPIA companies' in kind contribution]. German Federal Ministry of Education and Research [grant number 031A430B]; National Institutes of Health [grant number HL54732, HL098053, AG033456)].

\section{References}

1. Exner, N., Lutz, A.K., Haass, C. and Winklhofer, K.F. (2012) Mitochondrial dysfunction in parkinson's disease: Molecular mechanisms and pathophysiological consequences. embo J., 31, 3038-3062.

2. Schapira, A.H., Cooper, J.M., Dexter, D., Clark, J.B., Jenner, P. and Marsden, C.D. (1990) Mitochondrial complex I deficiency in parkinson's disease. J. Neurochem., 54, 823-827.

3. Schapira, A.H. (2007) Mitochondrial dysfunction in parkinson's disease. Cell Death Differ, 14, 1261-1266.

4. Parker, W.D., Jr, Boyson, S.J. and Parks, J.K. (1989) Abnormalities of the electron transport chain in idiopathic parkinson's disease. Ann. Neurol., 26, 719-723.

5. Muftuoglu, M., Elibol, B., Dalmizrak, O., Ercan, A., Kulaksiz, G., Ogus, H., Dalkara, T. and Ozer, N. (2004) Mitochondrial complex I and IV activities in leukocytes from patients with parkin mutations. Mov. Disord., 19, 544-548.

6. Mortiboys, H., Thomas, K.J., Koopman, W.J., Klaffke, S., Abou-Sleiman, P., Olpin, S., Wood, N.W., Willems, P.H., Smeitink, J.A., Cookson, M.R. et al. (2008) Mitochondrial function and morphology are impaired in parkin-mutant fibroblasts. Ann. Neurol., 64, 555-565.

7. Celardo, I., Martins, L.M. and Gandhi, S. (2013) Unravelling mitochondrial pathways to parkinson's disease. Br. J. Pharmacol., 171, 1943-1957. 
8. Rana, A., Rera, M. and Walker, D.W. (2013) Parkin overexpression during aging reduces proteotoxicity, alters mitochondrial dynamics, and extends lifespan. Proc. Natl. Acad. Sci. U. S. A, 110, 8638-8643.

9. Rothfuss, O., Fischer, H., Hasegawa, T., Maisel, M., Leitner, P., Miesel, F., Sharma, M., Bornemann, A., Berg, D., Gasser, T. et al. (2009) Parkin protects mitochondrial genome integrity and supports mitochondrial DNA repair. Hum. Mol. Genet., 18, 3832-3850.

10. Greene, J.C., Whitworth, A.J., Kuo, I., Andrews, L.A., Feany, M.B. and Pallanck, L.J. (2003) Mitochondrial pathology and apoptotic muscle degeneration in drosophila parkin mutants. Proc. Natl. Acad. Sci. U. S. A, 100, 4078-4083.

11. Palacino, J.J., Sagi, D., Goldberg, M.S., Krauss, S., Motz, C., Wacker, M., Klose, J. and Shen, J. (2004) Mitochondrial dysfunction and oxidative damage in parkin-deficient mice. J. Biol. Chem., 279, 18614-18622.

12. Flinn, L., Mortiboys, H., Volkmann, K., Koster, R.W., Ingham, P.W. and Bandmann, O. (2009) Complex I deficiency and dopaminergic neuronal cell loss in parkin-deficient zebrafish (danio rerio). Brain, 132, 1613-1623.

13. Grunewald, A., Voges, L., Rakovic, A., Kasten, M., Vandebona, H., Hemmelmann, C., Lohmann, K., Orolicki, S., Ramirez, A., Schapira, A.H. et al. (2010) Mutant parkin impairs mitochondrial function and morphology in human fibroblasts. PLoS One, 5, e12962.

14. Narendra, D., Tanaka, A., Suen, D.F. and Youle, R.J. (2008) Parkin is recruited selectively to impaired mitochondria and promotes their autophagy. J. Cell Biol., 183, 795-803.

15. Vives-Bauza, C., Zhou, C., Huang, Y., Cui, M., de Vries, R.L., Kim, J., May, J., Tocilescu, M.A., Liu, W., Ko, H.S. et al. (2010) PINK1-dependent recruitment of parkin to mitochondria in mitophagy. Proc. Natl. Acad. Sci. U. S. A, 107, 378-383.

16. Rakovic, A., Grunewald, A., Seibler, P., Ramirez, A., Kock, N., Orolicki, S., Lohmann, K. and Klein, C. (2010) Effect of endogenous mutant and wild-type PINK1 on parkin in fibroblasts from parkinson disease patients. Hum. Mol. Genet., 19, 3124-3137.

17. Chan, N.C., Salazar, A.M., Pham, A.H., Sweredoski, M.J., Kolawa, N.J., Graham, R.L., Hess, S. and Chan, D.C. (2011) Broad activation of the ubiquitin-proteasome system by parkin is critical for mitophagy. Hum. Mol. Genet., 20, 1726-1737.

18. Hajek, P., Chomyn, A. and Attardi, G. (2007) Identification of a novel mitochondrial complex containing mitofusin 2 and stomatin-like protein 2. J. Biol. Chem., 282, 5670-5681.

19. Christie, D.A., Lemke, C.D., Elias, I.M., Chau, L.A., Kirchhof, M.G., Li, B., Ball, E.H., Dunn, S.D., Hatch, G.M. and Madrenas, J. (2011) Stomatin-like protein 2 binds cardiolipin and regulates mitochondrial biogenesis and function. Mol. Cell. Biol., 31, 3845-3856.

20. Zanon, A., Rakovic, A., Blankenburg, H., Doncheva, N.T., Schwienbacher, C., Serafin, A., Alexa, A., Weichenberger, C.X., Albrecht, M., Klein, C. et al. (2013) Profiling of parkinbinding partners using tandem affinity purification. PLoS One, 8, e78648.

21. Christie, D.A., Mitsopoulos, P., Blagih, J., Dunn, S.D., StPierre, J., Jones, R.G., Hatch, G.M. and Madrenas, J. (2012) Stomatin-like protein 2 deficiency in $\mathrm{T}$ cells is associated with altered mitochondrial respiration and defective CD4+ $\mathrm{T}$ cell responses. J. Immunol., 189, 4349-4360.

22. Tondera, D., Grandemange, S., Jourdain, A., Karbowski, M., Mattenberger, Y., Herzig, S., Da Cruz, S., Clerc, P., Raschke, I., Merkwirth, C. et al. (2009) SLP-2 is required for stressinduced mitochondrial hyperfusion. embo J., 28, 1589-1600.
23. Gegg, M.E., Cooper, J.M., Chau, K.Y., Rojo, M., Schapira, A.H. and Taanman, J.W. (2010) Mitofusin 1 and mitofusin 2 are ubiquitinated in a PINK1/parkin-dependent manner upon induction of mitophagy. Hum. Mol. Genet., 19, 4861-4870.

24. Rakovic, A., Grunewald, A., Kottwitz, J., Bruggemann, N., Pramstaller, P.P., Lohmann, K. and Klein, C. (2011) Mutations in PINK1 and parkin impair ubiquitination of mitofusins in human fibroblasts. PLoS One, 6, e16746.

25. Betzer, C., Movius, A.J., Shi, M., Gai, W.P., Zhang, J. and Jensen, P.H. (2015) Identification of synaptosomal proteins binding to monomeric and oligomeric alpha-synuclein. PLoS One, 10, e0116473.

26. Lutz, A.K., Exner, N., Fett, M.E., Schlehe, J.S., Kloos, K., Lammermann, K., Brunner, B., Kurz-Drexler, A., Vogel, F., Reichert, A.S. et al. (2009) Loss of parkin or PINK1 function increases Drp1-dependent mitochondrial fragmentation. J. Biol. Chem., 284, 22938-22951.

27. Cali, T., Ottolini, D., Negro, A. and Brini, M. (2013) Enhanced parkin levels favor ER-mitochondria crosstalk and guarantee $\mathrm{ca}(2+)$ transfer to sustain cell bioenergetics. Biochim. Biophys. Acta, 1832, 495-508.

28. Chung, S.Y., Kishinevsky, S., Mazzulli, J.R., Graziotto, J., Mrejeru, A., Mosharov, E.V., Puspita, L., Valiulahi, P., Sulzer, D., Milner, T.A. et al. (2016) Parkin and PINK1 patient iPSC-derived midbrain dopamine neurons exhibit mitochondrial dysfunction and alpha-synuclein accumulation. Stem Cell. Reports, 7, 664-677.

29. Morais, V.A., Haddad, D., Craessaerts, K., De Bock, P.J., Swerts, J., Vilain, S., Aerts, L., Overbergh, L., Grunewald, A., Seibler, P. et al. (2014) PINK1 loss-of-function mutations affect mitochondrial complex I activity via NdufA10 ubiquinone uncoupling. Science, 344, 203-207.

30. Pramstaller, P.P., Schlossmacher, M.G., Jacques, T.S., Scaravilli, F., Eskelson, C., Pepivani, I., Hedrich, K., Adel, S., Gonzales-McNeal, M., Hilker, R. et al. (2005) Lewy body parkinson's disease in a large pedigree with 77 parkin mutation carriers. Ann. Neurol., 58, 411-422.

31. Hedrich, K., Kann, M., Lanthaler, A.J., Dalski, A., Eskelson, C., Landt, O., Schwinger, E., Vieregge, P., Lang, A.E., Breakefield, X.O. et al. (2001) The importance of gene dosage studies: Mutational analysis of the parkin gene in early-onset parkinsonism. Hum. Mol. Genet., 10, 1649-1656.

32. Klein, C., Pramstaller, P.P., Kis, B., Page, C.C., Kann, M., Leung, J., Woodward, H., Castellan, C.C., Scherer, M., Vieregge, P. et al. (2000) Parkin deletions in a family with adult-onset, tremor-dominant parkinsonism: Expanding the phenotype. Ann. Neurol., 48, 65-71.

33. Park, J., Kim, Y. and Chung, J. (2009) Mitochondrial dysfunction and parkinson's disease genes: Insights from drosophila. Dis. Model. Mech., 2, 336-340.

34. Guo, M. (2012) Drosophila as a model to study mitochondrial dysfunction in parkinson's disease. Cold Spring Harb. Perspect. Med., 2, 10.1101/cshperspect.a009944.

35. Sen, A., Kalvakuri, S., Bodmer, R. and Cox, R.T. (2015) Clueless, a protein required for mitochondrial function, interacts with the PINK1-parkin complex in drosophila. Dis. Model. Mech., 8, 577-589.

36. Liu, W., Acin-Perez, R., Geghman, K.D., Manfredi, G., Lu, B. and Li, C. (2011) Pink1 regulates the oxidative phosphorylation machinery via mitochondrial fission. Proc. Natl. Acad. Sci. U. S. A, 108, 12920-12924.

37. Clark, I.E., Dodson, M.W., Jiang, C., Cao, J.H., Huh, J.R., Seol, J.H., Yoo, S.J., Hay, B.A. and Guo, M. (2006) Drosophila pink1 is required for mitochondrial function and interacts genetically with parkin. Nature, 441, 1162-1166. 
38. Lees, A.J., Hardy, J. and Revesz, T. (2009) Parkinson's disease. Lancet, 373, 2055-2066.

39. Ghose, P., Park, E.C., Tabakin, A., Salazar-Vasquez, N. and Rongo, C. (2013) Anoxia-reoxygenation regulates mitochondrial dynamics through the hypoxia response pathway, SKN-1/nrf, and stomatin-like protein STL-1/SLP-2. PLOS Genet., 9, e1004063.

40. Mitsopoulos, P., Chang, Y.H., Wai, T., Konig, T., Dunn, S.D., Langer, T. and Madrenas, J. (2015) Stomatin-like protein-2 is required for in vivo mitochondrial respiratory chain supercomplex formation and optimal cell function. Mol. Cell. Biol., 35, 1838-1847.

41. Meka, D.P., Muller-Rischart, A.K., Nidadavolu, P., Mohammadi, B., Motori, E., Ponna, S.K., Aboutalebi, H., Bassal, M., Annamneedi, A., Finckh, B. et al. (2015) Parkin cooperates with GDNF/RET signaling to prevent dopaminergic neuron degeneration. J. Clin. Invest., 125, 1873-1885.

42. Imaizumi, Y., Okada, Y., Akamatsu, W., Koike, M., Kuzumaki, N., Hayakawa, H., Nihira, T., Kobayashi, T., Ohyama, M., Sato, S. et al. (2012) Mitochondrial dysfunction associated with increased oxidative stress and alphasynuclein accumulation in PARK2 iPSC-derived neurons and postmortem brain tissue. Mol. Brain, 5, 35-6606-5-35.

43. Exner, N., Treske, B., Paquet, D., Holmstrom, K., Schiesling, C., Gispert, S., Carballo-Carbajal, I., Berg, D., Hoepken, H.H., Gasser, T. et al. (2007) Loss-of-function of human PINK1 results in mitochondrial pathology and can be rescued by parkin. J. Neurosci., 27, 12413-12418.

44. Ali, Y.O., Escala, W., Ruan, K. and Zhai, R.G. (2011) Assaying locomotor, learning, and memory deficits in drosophila models of neurodegeneration. J. Vis. Exp., 49, pii: 2504. doi, 10.3791/2504.

45. Muller-Rischart, A.K., Pilsl, A., Beaudette, P., Patra, M., Hadian, K., Funke, M., Peis, R., Deinlein, A., Schweimer, C., Kuhn, P.H. et al. (2013) The E3 ligase parkin maintains mitochondrial integrity by increasing linear ubiquitination of NEMO. Mol. Cell, 49, 908-921.

46. Wai, T., Saita, S., Nolte, H., Muller, S., Konig, T., RichterDennerlein, R., Sprenger, H.G., Madrenas, J., Muhlmeister, M., Brandt, U. et al. (2016) The membrane scaffold SLP2 anchors a proteolytic hub in mitochondria containing PARL and the i-AAA protease YME1L. EMBO Rep., 17, 1844-1856.

47. Acin-Perez, R., Fernandez-Silva, P., Peleato, M.L., PerezMartos, A. and Enriquez, J.A. (2008) Respiratory active mitochondrial supercomplexes. Mol. Cell, 32, 529-539.

48. Vincow, E.S., Merrihew, G., Thomas, R.E., Shulman, N.J., Beyer, R.P., MacCoss, M.J. and Pallanck, L.J. (2013) The PINK1parkin pathway promotes both mitophagy and selective respiratory chain turnover in vivo. Proc. Natl. Acad. Sci. U. S. A, 110, 6400-6405.

49. Seibler, P., Graziotto, J., Jeong, H., Simunovic, F., Klein, C. and Krainc, D. (2011) Mitochondrial parkin recruitment is impaired in neurons derived from mutant PINK1 induced pluripotent stem cells. J. Neurosci., 31, 5970-5976.

50. Xu, C., Inokuma, M.S., Denham, J., Golds, K., Kundu, P., Gold, J.D. and Carpenter, M.K. (2001) Feeder-free growth of undifferentiated human embryonic stem cells. Nat. Biotechnol., 19, 971-974.

51. Kriks, S., Shim, J.W., Piao, J., Ganat, Y.M., Wakeman, D.R., Xie, Z., Carrillo-Reid, L., Auyeung, G., Antonacci, C., Buch, A. et al. (2011) Dopamine neurons derived from human ES cells efficiently engraft in animal models of parkinson's disease. Nature, 480, 547-551.

52. Stanslowsky, N., Haase, A., Martin, U., Naujock, M., Leffler, A., Dengler, R. and Wegner, F. (2014) Functional differentiation of midbrain neurons from human cord blood-derived induced pluripotent stem cells. Stem Cell. Res. Ther., 5, 35.

53. Thymiakou, E. and Episkopou, V. (2011) Detection of signaling effector-complexes downstream of bmp4 using PLA, a proximity ligation assay. J. Vis. Exp., 49, pii: 2631. doi, 10.3791/2631.

54. Park, I.H., Lerou, P.H., Zhao, R., Huo, H. and Daley, G.Q. (2008) Generation of human-induced pluripotent stem cells. Nat. Protoc., 3, 1180-1186.

55. Almeida, A. and Medina, J.M. (1997) Isolation and characterization of tightly coupled mitochondria from neurons and astrocytes in primary culture. Brain Res., 764, 167-172.

56. Darley-Usmar, V., Rickwood, D. and Wilson, M. (1987) Subfractionation of Mitochondria and Isolation of the Proteins of Oxidative Phosphorylation. London, IRL Press.

57. Coore, H.G., Denton, R.M., Martin, B.R. and Randle, P.J. (1971) Regulation of adipose tissue pyruvate dehydrogenase by insulin and other hormones. Biochem. J., 125, 115-127.

58. Liu, S. and Lu, B. (2010) Reduction of protein translation and activation of autophagy protect against PINK1 pathogenesis in drosophila melanogaster. PLoS Genet., 6, e1001237.

59. Choo, Y.S., Vogler, G., Wang, D., Kalvakuri, S., Iliuk, A., Tao, W.A., Bodmer, R. and Zhang, Z. (2012) Regulation of parkin and PINK1 by neddylation. Hum. Mol. Genet., 21, 2514-2523.

60. Wells, L., Edwards, K.A. and Bernstein, S.I. (1996) Myosin heavy chain isoforms regulate muscle function but not myofibril assembly. embo J., 15, 4454-4459.

61. Barone, M.C. and Bohmann, D. (2013) Assessing neurodegenerative phenotypes in drosophila dopaminergic neurons by climbing assays and whole brain immunostaining. J. Vis. Exp., 74, e50339. doi, e50339.

62. Wu, Z., Sawada, T., Shiba, K., Liu, S., Kanao, T., Takahashi, R., Hattori, N., Imai, Y. and Lu, B. (2013) Tricornered/NDR kinase signaling mediates PINK1-directed mitochondrial quality control and tissue maintenance. Genes Dev., 27, 157-162.

63. Tukey, J.W. (1977) Exploratory Data Analysis. Reading, Massachusetts. Addison Wesley Publishing Company.

64. Snijders, T. (1999) Multilevel Analysis: An Introduction to Basic and Advanced Multilevel Modeling. London SAGE Publications Inc. 